\title{
Hepatocellular Carcinoma Occurrence and Recurrence in Hepatitis C-infected Patients Treated with Direct-acting Antivirals
}

Taseen Syed ${ }^{1}$, Javid Fazili Dr. ${ }^{2}$, Ijlal Akbar Ali ${ }^{3}$, Daniel Zhao ${ }^{4}$, Diane Hughes ${ }^{2}$, Sultan Mahmood MD 5

1. Internal Medicine, University of Oklahoma Health Sciences Center, Oklahoma City, USA 2. Section of Digestive Diseases \& Nutrition, University of Oklahoma Health Sciences Center, Oklahoma City, USA 3. Section of Digestive Diseases and Nutrition, University of Oklahoma Health Sciences Center, Oklahoma City, USA 4. Biostatistics, University of Oklahoma Health Sciences Center, Oklahoma City, USA 5. Gastroenterology Fellow Section of Digestive Diseases \& Nutrition, University of Oklahoma Health Sciences Center, Oklahoma City, USA

$\square$ Corresponding author: Taseen Syed, taseen-syed@ouhsc.edu Disclosures can be found in Additional Information at the end of the article

\section{Abstract}

Introduction

Multiple studies have shown the efficacy of the new direct-acting antivirals (DAAs) with a cure rate of over $90 \%$ in hepatitis C virus (HCV)-infected patients. Some recently published studies have suggested an increased incidence of de novo and recurrent hepatocellular carcinoma (HCC) in cirrhotic patients in sustained virological response (SVR) after completing therapy. A possible mechanism is the breakdown of immune surveillance after starting DAAs. We report a retrospective analysis on a population of chronic HCV infected patients, with and without a prior history of HCC, who developed HCC after receiving DAAs in the hope of adding to existing literature and in pursuit of greater clarity into this emerging concern with DAAs.

Methods

We analyzed 497 HCV-infected patients who were treated with DAAs, or a combination of DAA with interferon, from January 2014 to April 2017 at the Veterans Medical Center, Oklahoma City. Descriptive analysis, including the mean and standard deviation for different variables, was used. The cohort was divided into two groups: cirrhotic and non-cirrhotic. The analysis was run in the cirrhotic group between the subgroups who developed HCC and who did not.

Results

Received 05/16/2018 Review began 05/29/2018 Review ended 06/14/2018 Published 06/19/2018

\section{C) Copyright 2018}

Syed et al. This is an open access article distributed under the terms of the Creative Commons Attribution License CC-BY 3.0., which permits unrestricted use, distribution, and reproduction in any medium, provided the original author and source are credited.
Data from a total of 233 cirrhotic patients were analyzed. We further subdivided these patients into those who eventually were diagnosed with HCC (group 1) and those who were not (group 2). These subgroups were comparable in regards to race, gender, baseline serum aspartate aminotransferase (AST), alanine aminotransferase (ALT), platelets, sodium, HCV genotypes, and pretreatment viral load. All patients completed therapy. The rate of SVR was much lower in group 1 compared to group 2 (62.5\% vs 88.94\%, $\mathrm{p}=0.002)$, respectively. Model End-stage Liver Disease (MELD) score, Child-Turcotte-Pugh (CTP) score, and Fibrosis-4 (FIB-4) score were higher in the group that developed HCC. The average time period (weeks) from DAA therapy to HCC diagnosis was 48.2 weeks. The remaining 264 non-cirrhotic patients had no reported cases of HCC. 


\section{Cureus}

Conclusion

From a total of 497 treated HCV-infected patients, 233 (46.88 \%) had cirrhosis, out of which 16 (6.86\%) were reported to develop HCC during or after DAA therapy was initiated. The remaining 217 (93.1\%) cirrhotic patients did not develop HCC. As per our comparison, achieving SVR in cirrhotic patients should not preclude HCC screening, and more studies are needed to assess the risk of HCC in patients who achieve SVR but have a high FIB-4 score. In fact, patients who do not achieve SVR may be at a higher risk of eventually developing HCC and may be candidates for closer surveillance.

Categories: Internal Medicine, Gastroenterology, Infectious Disease

Keywords: hepatitis c, direct-acting antivirals (daa), sustained virological response

\section{Introduction}

Multiple recent studies have suggested an increased incidence of de novo and recurrent hepatocellular carcinoma (HCC) in cirrhotic patients with a sustained virologic response (SVR) after completing therapy with direct-acting antivirals (DAAs) [1-4]. Recent studies also suggest lower SVR rates in patients with hepatitis C virus (HCV)-related cirrhosis and underlying HCC when compared to patients with only HCV cirrhosis [5]. In the hope of adding to the literature, we performed a retrospective analysis to assess the rates of SVR and the incidence and recurrence of HCC in chronic HCV-infected patients who were treated with DAAs at our institution.

\section{Materials And Methods}

We analyzed 497 HCV-infected patients who were treated with DAAs, or a combination of DAA with interferon, from January 2014 to April 2017 at the Veterans Medical Center, Oklahoma City. Descriptive analysis, including the mean and standard deviation for different variables, was used. The cohort was divided into two groups: cirrhotic and non-cirrhotic. The analysis was run in the cirrhotic group between the subgroups who developed HCC and who did not. A P value $<0.05$ was considered statistically significant.

\section{Results}

Two hundred and thirty-three patients (46.88 \%) had cirrhosis, of which 16 (6.86\%) developed HCC (11 de novo and five recurrent cases) during or after DAA therapy was initiated, whilst the remaining 217 (93.1\%) cirrhotic patients did not develop HCC. None of the non-cirrhotic patients developed HCC. We further subdivided the cirrhotic group into those who eventually were diagnosed with HCC (group 1) and those who were not (group 2). These subgroups were comparable in regards to race, gender, baseline serum aspartate aminotransferase (AST), alanine aminotransferase (ALT), platelets, sodium, HCV genotypes, and pretreatment viral load. All patients completed therapy. The rate of SVR was much lower in group 1 compared to group $2(62.5 \%$ vs $88.94 \%, p=0.002)$, respectively. The Fibrosis-4 (FIB-4) score was higher in group 1 as compared to group $2(7.88$ vs $4.09, \mathrm{p}=0.048)$, respectively. The average time period (weeks) from completion of DAA therapy to HCC diagnosis was 48.2 weeks and from time of SVR to HCC detection was 33.3 weeks (Table 1).

\section{Variables}

AGE, average
HCV treated cirrhotic patients

with HCC ( $n=16)$

66.19
HCV treated cirrhotic patients without HCC ( $=217)$

62.09
P. Value

0.005 


\section{Cureus}

Male sex, n (\%) [number \& percentage of patients]

RACE

a. White, n (\%)

b. AA, n (\%)

c. Other, n (\%)

a. Genotype 1a

9 (56.25)

b. Genotype 1b

$3(18.75)$

$2(12.5)$

c. Genotype 2

d. Genotype 3

History of alcohol, n (\%)

$2(12.5)$

$10(62.5)$

$11(68.75)$

History of smoking, n (\%)

$5(31.25)$

History of IV drug use, n (\%)

$1,286,176$

Mean Value

Patients achieving SVR, n (\%)

$10(62.5)$

MELD Score, mean (median) at SVR

11.31(11)

$6.31(6)$

7.88 (6.52)

$4.98(2.96)$

0.048

(median)

AST at SVR, mean (median)

$62.81(48)$

$40.33(28)$

0.104

ALT at SVR, mean (median)

$45.75(29)$

$31.55(23)$

0.201

Hemoglobin at SVR, mean

(median)

13.06 (13.05)

14.09 (14.5)

0.045

T. Bilirubin at SVR, mean (median)

1.53 (1.3)

$1.03(0.8)$

0.005

Albumin at SVR, mean (median)

$\mathrm{Na}$ at SVR

3.23 (3.2)

3.73 (3.8)

$<0.001$

136.69 (137)

142.04 (137)

0.758

108.75(96.5)

Treatment naïve/Non-Treatment Naïve, n (\%) 


\section{Cureus}
a. Treatment naïve, $\mathrm{n}(\%)$
$6(37.5)$
$155(71.43)$
b. Non-Treatment naïve, $n(\%)$
$10(62.5)$
$62(28.57)$

Treatment regimen, $\mathrm{n}(\%)$

a. DAA only based regimen, $n$ (\%) $13(81.25)$

207 (95.39)

b. DAA \& IFN based regimens, $\mathrm{n}$

(\%)

c. Use of Ribavirin, $n$ (\%)

115 (52.99)

Comorbidities due to Chronic

Liver Disease

a. Ascites, $\mathrm{n}(\%)$

$2(12.5)$

$14(6.48)$

0.359

b. Esophageal Varices, $\mathrm{n}(\%)$

$8(50)$

$47(21.66)$

\section{TABLE 1: Table: Comparative analysis between cirrhotic patients with and without hepatocellular carcinoma.}

Demographic and laboratory variables along with outcome assessment with Chi-Square Test or T-test. Acronyms: Hepatocellular carcinoma (HCC), Hepatitis C Virus (HCV), Aferican American (AA), Model for End-Stage Liver Disease (MELD), Child-TurcottePugh (CTP), Fibrosis-4 (FIB-4) Score, sustained virological response (SVR), Interfron (IFN), and direct acting antiviral (DAA).

\section{Discussion}

According to the Centers for Disease Control (CDC), of patients infected with hepatitis C, 5\% $20 \%$ develop cirrhosis over $20-30$ years. Of these, $1 \%-5 \%$ die due to decompensated cirrhosis or HCC. Unadjusted data from a study conducted over a course of a decade (2001 - 2013) in United States veterans, published in November 2015, showed an incidence of HCC increased by $265 \%$, mortality in cirrhotics increased by $51 \%$ and by $285 \%$ in patients with HCC [6]. With this global burden of hepatitis C, DAA-based regimens, with high efficacy and optimal safety profiles, are the best treatment option to date. However, recent studies have questioned the safety profile of these DAAs [2, 7-8]. A proposed hypothesis is the disruption of immune surveillance after starting DAA-based antiviral therapy [3]. At the other end of the spectrum, analysis of three French prospective multicenter cohorts, a prospective observational study, and analysis of a retrospective cohort of HCV-infected veterans treated with DAAs all showed no increase in HCC recurrence in patients treated with DAAs [4, 9-10]. Recent studies also demonstrate a lower SVR in HCV cirrhotics with underlying HCC. There are numerous proposed mechanisms for this, including HCC acting as a reservoir for HCV replication, distorted liver architecture due to HCC, lack of delivery of DAAs into HCC, different resistant strains of HCV infected patients with HCC, and immune dysregulation [5].

\section{Conclusions}

As per our comparison, achieving SVR in cirrhotic patients should not preclude HCC screening and more studies are needed to assess the risk of HCC in patients who achieve SVR but have a high FIB-4 Score. In fact, patients who do not achieve SVR, may be at a higher risk of eventually developing HCC, and may be candidates for closer surveillance. Affirmative conclusions cannot be drawn based on the nature of all studies to date but it reinforces the need for statistically 
stronger studies on larger cohorts that takes into account all the flaws of previous studies. In this current era of questionable association of DAAs with HCC, ongoing HCC surveillance in cirrhotics achieving SVR from DAAs is a reasonable approach until we get further clarification.

This work was presented as poster presentation at Digestive Disease Week 2018.

\section{Additional Information}

\section{Disclosures}

Human subjects: Consent was obtained by all participants in this study. Institutional Review Board, VA Medical Center, Oklahoma City, Oklahoma issued approval 8265. This was a retrospective chart review of human subjects who were treated for hepatitis $\mathrm{C}$. There was no direct contact with potential participants. No risks to subjects as study was retrospective chart review. All potential identifiers have been removed. This study was approved by VA Medical Center, Oklahoma City. Animal subjects: All authors have confirmed that this study did not involve animal subjects or tissue. Conflicts of interest: In compliance with the ICMJE uniform disclosure form, all authors declare the following: Payment/services info: All authors have declared that no financial support was received from any organization for the submitted work. Financial relationships: All authors have declared that they have no financial relationships at present or within the previous three years with any organizations that might have an interest in the submitted work. Other relationships: All authors have declared that there are no other relationships or activities that could appear to have influenced the submitted work.

\section{References}

1. Reig M, Boix L: Bruix J. The impact of direct antiviral agents on the development and recurrence of hepatocellular carcinoma. Liver international : official journal of the International Association for the Study of the Liver. 2017;37, 1:136-9. 10.1111/liv.13321

2. Reig M, Marino Z, Perello C, Inarrairaegui M, Ribeiro A, Lens S: et al. Unexpected high rate of early tumor recurrence in patients with HCV-related HCC undergoing interferon-free therapy. Journal of. hepatology, 2016:719-26. 10.1016/j.jhep.2016.04.008

3. Villani R, Facciorusso A, Bellanti F, Tamborra R, Piscazzi A, Landriscina M: et al. DAAs Rapidly Reduce Inflammation but Increase Serum VEGF Level: A Rationale for Tumor Risk during Anti-HCV Treatment. PloS one. 2016;11(12, 0167934. 10.1371/journal.pone.0167934

4. Lack of evidence of an effect of direct-acting antivirals on the recurrence of hepatocellular carcinoma: Data from three ANRS cohorts. Journal of. hepatology, 2016:734-40. 10.1016/j.jhep.2016.05.045

5. Kushner T, Dieterich D: Saberi B. Direct-acting antiviral treatment for patients with hepatocellular carcinoma. Curr Opin Gastroenterol. 2018, 10.1097/MOG.0000000000000431

6. Beste LA, Leipertz SL, Green PK, Dominitz JA, Ross D: Ioannou GN. Trends in burden of cirrhosis and hepatocellular carcinoma by underlying liver disease in US veterans, 2001-2013. Gastroenterology. 2015;149(6. 1471-82.e5, 17-8. 10.1053/j.gastro.2015.07.056

7. Conti F, Buonfiglioli F, Scuteri A, Crespi C, Bolondi L, Caraceni P: et al. Early occurrence and recurrence of hepatocellular carcinoma in HCV-related cirrhosis treated with direct-acting antivirals. Journal of. hepatology, 2016:727-33. 10.1016/j.jhep.2016.06.015

8. Yang JD, Aqel BA, Pungpapong S, Gores GJ, Roberts LR: Leise MD. Direct acting antiviral therapy and tumor recurrence after liver transplantation for hepatitis C-associated hepatocellular carcinoma. Journal of. hepatology, 2016:859-60. 10.1016/j.jhep.2016.06.023

9. Torres HA, Vauthey JN, Economides MP, Mahale P: Kaseb A. Hepatocellular carcinoma recurrence after treatment with direct-acting antivirals: First, do no harm by withdrawing treatment. Journal of. hepatology, 2016:862-4. 10.1016/j.jhep.2016.05.034

10. Kanwal F, Kramer J, Asch SM, Chayanupatkul M, Cao Y: El-Serag HB. Risk of Hepatocellular Cancer in HCV Patients Treated With Direct-Acting Antiviral Agents. Gastroenterology. 2017;153(4, 996:1. 10.1053/j.gastro.2017.06.012 